\title{
AN ELEMENTARY PROOF OF THE STRONG FORM OF THE CAUCHY THEOREM
}

\author{
LYNN H. LOOMIS
}

A difficult step in the derivation of the strong forms of the Cauchy theorem, Green's lemma, and related theorems from the corresponding weak forms is the construction, for a given rectifiable Jordan curve $J$, of a sequence of Jordan polygons lying interior to $J$, converging to $J$, and having uniformly bounded lengths. This note presents what the author believes to be a simpler elementary construction of this sequence than any hitherto available (see $[1,2]^{1}$ and the bibliographies at the ends of these papers). We shall illustrate its use by proving the strong Cauchy theorem.

We assume the Jordan separation theorem together with its elementary consequences, as is usual in proofs of this kind, and we assume the weak form of the Cauchy theorem $\left(\int_{P} f(z) d z=0\right.$ if $f(z)$ is analytic in a region containing $P$ and its interior) for Jordan polygons $P$ whose edges lie on lines of the form $x=m 2^{-N}, y=n 2^{-N}$, where $m, n=0, \pm 1, \cdots$. The polygon $P$ and its interior is of course the sum of a finite number of squares of the network of closed squares (with sides $2^{-N}$ ) into which these lines cut up the plane, so that we need to assume the weak Cauchy theorem only for single squares.

Let $X$ be a fixed interior point of the given rectifiable Jordan curve $J$ with coordinates not of the form $m 2^{-N}$, and let $C$ be a fixed closed square lying interior to $J$, containing $X$ and having sides parallel to the axes. Let $I$ be the set of squares of the above network lying interior to $J$; for sufficiently large $N$ every square of $I$ which contains a point of $C$ lies interior to $J$. The vertical ray from $X$ cuts a first edge $l_{1}$ of a square not in $I$. We form a polygon $P$ by proceeding to the left along $l_{1}$ from its right-hand end point $p_{0}$, and at any vertex $p_{n}$ choosing as $l_{n+1}$ from the three remaining edges the counter-clockwisemost one which has a square of $I$ on its left and a square not in $I$ (and so containing a point of $J$ ) on its right. We shall call the latter square $S_{n+1}$. The reader will find the following argument trivial if he will sketch the four possible configurations at $p_{n}$. In tracing $P$ a first vertex $p_{n}$ must be repeated, $p_{n}=p_{n+M}$. If $n>0$, then $l_{n}$ and $l_{n+M}$ approach $p_{n}$ from opposite directions, $S_{n+1}=S_{n+M}$, and the Jordan polygon $l_{n+1}$ to $l_{n+M}$ separates $S_{n+1}$ from $S_{n}$ and so separates points of $J$; an impossibility. Thus $n=0, p_{M}=p_{0}$ and $P$ is a Jordan polygon. Note

Received by the editors May 5, 1944.

1 Numbers in brackets refer to the bibliography at the end of the paper. 
that $P$ depends on $N$, although it is not explicit in the notation.

Let $q_{n}$ be a point of $J$ in $S_{n}$ nearest to $l_{n}$, and let $q_{n} t_{n}$ be the perpendicular dropped from $q_{n}$ to $l_{n}\left(t_{n}\right.$ on $\left.l_{n}\right)$. Because of the definition of the $q$ 's no two distinct segments $q_{n} t_{n}$ and $q_{m} t_{m}$ can intersect except possibly in a common end point; in particular, the order of the $t_{n}$ on $P$ is the same as that of the $q_{n}$ on $J$.

As a general remark, the squares touching any $S_{n}$ have a total of 24 edges, so that, for some $k \leqq 24, S_{n+k}$ does not touch $S_{n}$ and the length of the arc $q_{n} q_{n+k}$ of $J$ is at least $2^{-N}$. Thus if indices $n_{i}$ are chosen so that $24 \leqq n_{i+1}-n_{i}<48$ for $i=0, \cdots, K-1$ and $t_{n_{0}}=t_{n_{K}}=t_{0}$, then the length of the arc $q_{n_{i}} q_{n_{i+1}}$ of $J$ is at least $2^{-N}$ and the length of the arc $t_{n_{i}} t_{n_{i+1}}$ of $P$ is at most $48 / 2^{N}$. If $C_{i}$ is the Jordan curve consisting of these two arcs and the two segments $q_{n_{i}} t_{n_{i}}, q_{n_{i+1}} t_{n_{i+1}}$, we have that $L\left(C_{i}\right) \leqq 51 \cdot L\left(q_{n_{i}} q_{n_{i+1}}\right)$, and

$$
\sum_{i=0}^{K-1} L\left(C_{i}\right) \leqq 51 \cdot L(J) .
$$

We can now apply the device of Newman $[3$, p. 156] to prove the strong Cauchy theorem.

TheOREM. If $f(z)$ is analytic interior to $J$ and continuous on $J$ plus its interior, then $\int_{J} f(z) d z=0$.

The distance from $q_{n_{i}}$ to $q_{n_{i+1}}$ is for every $i$ at most $50 / 2^{N}$, and since $J$ is rectifiable it follows that given $\delta$ there is an $N$ such that for every $i$ one of the two arcs determined on $J$ by $q_{n_{i}}$ and $q_{n_{i}+1}$ has length at most $\delta$. But this must be the arc we have called $q_{n_{i}} q_{n_{i+1}}$ (containing $q_{j}$ for $n_{i} \leqq j \leqq n_{i+1}$ ); for the length of its complementary arc on $J$ is at least $1 / 48$ th of the length of the major arc of the points $t_{n_{i}}$ and $t_{n_{i+1}}$ on $P$, and since the square $C$ lies interior to $P$ this length is bounded below by one-half the length of the perimeter of $C$. Thus the maximum diameter of the curves $C_{i}$ approaches 0 as $N \rightarrow \infty$ and since $f(z)$ is uniformly continuous on $J$ plus its interior, there exists for every $\epsilon$ an $N$ such that $\left|f(z)-f\left(t_{n_{i}}\right)\right|<\epsilon$ for all $z$ on $C_{i}$. Now $\int_{J} f(z) d z=\sum_{0}^{K-1} \int_{C_{i}} f(z) d z$, for the difference between these two integrals consists of $\int_{P}$, which is 0 by hypothesis, and the integrals over the segments $p_{n_{i}} t_{n_{i}}$, which are traced once in each direction and so cancel. Since $\int_{C_{i}} f\left(t_{n_{i}}\right) d z=f\left(t_{n_{i}}\right) \int_{C_{i}} d z=0$, this implies that

$$
\left|\int_{J} f(z) d z\right|=\left|\sum_{i=0}^{K-1} \int_{C_{i}}\left(f(z)-f\left(t_{n_{i}}\right)\right) d z\right| \leqq \epsilon \cdot 51 L(J)
$$

for every $\epsilon$; thus $\int_{J} f(z) d z=0$. 


\section{BIBLIOGRAPHY}

1. M. R. Hestenes, An analogue of Green's theorem in the calculus of variations, Duke Math. J. vol. 8 (1941) pp. 300-311.

2. W. T. Reid, Green's lemma and related results, Amer. J. Math. vol. 63 (1941) pp. 563-574.

3. M. H. A. Newman, Elements of the topology of plane sets of points, Cambridge University Press, 1939.

HARVARD UNIVERSITY

\section{ON A CERTAIN TYPE OF NONLINEAR INTEGRAL EQUATIONS}

\section{MARK LOTKIN}

1. Introduction. The object of this paper is to prove that the nonlinear integral equation

$$
\phi(x)=\lambda\left[f(x)+\sum_{i=1}^{m} \int_{a}^{b} \cdots \int_{a}^{b} K_{i}\left(x, s_{1}, \cdots, s_{i}\right)\right.
$$

$$
\left.\cdot F_{i}\left(s_{1}, \cdots, s_{i}, \phi\left(s_{1}\right), \cdots, \phi\left(s_{i}\right)\right) d s_{1} \cdots d s_{i}\right]
$$

has at least one eigenvalue, provided the functionals

$$
\begin{aligned}
G_{i}(x, v)=\int_{a}^{b} \cdots \int_{a}^{b} K_{i}\left(x, s_{1}, \cdots, s_{i}\right) \\
\\
\qquad F_{i}\left(s_{1}, \cdots, s_{i}, v\left(s_{1}\right), \cdots, v\left(s_{i}\right)\right) d s_{1} \cdots d s_{i}
\end{aligned}
$$

are fully continuous, and the $F_{i}$ satisfy a certain linear integrodifferential equation. The solution of (1) is shown to be equivalent to that of a variational problem containing infinitely many parameters. The latter problem, however, can be solved easily by the method of Rayleigh-Ritz, which consists in approaching the solution of the variational problem by a sequence of variational problems containing only a finite number of parameters. The convergence of this procedure is assured by a convergence theorem of Friedrich Riesz.

2. Preparatory remarks. Let $I$ be the closed interval $a \leqq x \leqq b$, and $L^{2}$ the class of all functions having Lebesgue integrable squares on $I$ with a norm not larger than $N^{2}$. Let, further, $\left\{v_{n}(x)\right\}(n=1,2,3, \cdots)$

Received by the editors May 18, 1944. 